\title{
A flexible evolutionary model of machine learning of the most successful strategies of human capital development
}

\author{
Vasyl Porokhnya ${ }^{1}$ and Vladyslav Penev ${ }^{1, *}$ \\ ${ }^{1}$ Classical private university, 70B Zhukovskoho Str., Zaporizhzhia, 69002, Ukraine
}

\begin{abstract}
As a result of research, the concept of a flexible evolutionary model is proposed, which with the help of machine learning allows obtaining the most successful strategy for the development of human capital. The proposed conceptual and methodological approach to machine learning of the process of assessing human capital of enterprises, taking into account the cognitive psychology of man and reflective attitudes in the human environment, can increase the effectiveness of decision-making in the field of human capital development management. The training involves indicators of return on investment in the individual, in the types of components of human capital, which are characterized by properties (creativity, competence, purposefulness, communication, motivation), where between their varieties there are appropriate reflective relationships. The main difficulty of this approach to the choice of alternative solutions for finding options for the use of human capital is the correct selection of indicators of significance (return) of contributions to the development of types of human capital, on the basis of which cycles occur of systemic learning. This approach can simplify the search for and developments of human capital development strategies, present alternative ways, and simplify management decisions.
\end{abstract}

\section{Introduction}

Human capital (HC) in the modern economy is one of the most important components of an enterprise or state for economic growth. The article [1] uses the decision tree model is used to analyze the performance appraisal of hospital staff to explore the several important factors that affect performances. The purpose of the article [2] is to analyze how objective factors influence the departure of employees in order to determine the main reasons contributing to the decision of an employee to leave the company, as well as the ability to predict whether a particular employee will be able to leave the company. Unfortunately, machine learning for assessing the properties of human capital is in its early stages. Human capital has a strong impact on the competitiveness of the assessed entity. Although the use of funds to improve the value and quality of human capital is still considered to be partly costly rather than an investment, the decision to increase human capital is a hot topic of research, because decision-making in this area is associated with significant uncertainty about the characteristics of properties. and relations that form the concept of human capital. And first of all, there are natural signs of human behavior and their measurement: reflexive, cognitive. Therefore, the main thing is that we need to know the characteristics of human behavior. To know to what extent the process of assessing the development of human capital of an individual, enterprise, or state personnel coincides with the knowledge about a man that humanity possesses. To do this, it will be useful, in our opinion, to build a model

*e-mail: penev.vladislav@gmail.com for assessing human capital, which would be reflected in the mechanisms of machine learning to find alternatives to increase human capital.

In the framework of this study, the expediency of using machine learning to increase the reliability of obtaining the most effective strategy for human capital development is substantiated.

The study simulated strategies for human capital development using machine learning as a set of possible actions that differently affect the values of parameters that change the amount of human capital to increase its value. As a result of the study, the concept of a flexible model was proposed, which using machine learning allows obtaining the most successful alternative to the development of human capital.

\section{Results}

If we consider the structure of human capital as a set of qualities of its properties, their ratios, which directly affect productivity, which increases income for staff, the company as a whole, society, nation, it is possible to cover all possible options for its evaluation. It should be said that human capital is a form of capital that is able to generate income that is valued with the influence of natural factors inherent only in man.

The power of the impact of human capital on the competitiveness of the enterprise exacerbates the interest of entrepreneurs and the state to increase the human capital of individuals, to make it more efficient. Thus, Simon Smith Kuznets, introducing the concept of GDP, noted that one 
of the necessary factors for the development of the country (including increasing its GDP) is the growth of human capital.

In order to assess the impact of the HC of a company, organization, enterprise on its financial results, it is necessary that it has a quantitative assessment. Since the calculation of financial indicators is mainly based on costs, it is optimal to take as a basis for the quantitative assessment of the human capital cost approach, taking into account the cognitive psychology of man and reflexive relations in the system of HC figure 1.

The advantage of the cost approach to the evaluation of human capital is that it allows you to get the amount of $\mathrm{HC}$ in the valuation, integrated with estimates of other sources, such as fixed, working capital, organizational, and consumer capital $[3,4]$.

The structure of $\mathrm{HC}$ costs is primarily related to staff remuneration. Of course, these are payments to employees, including wages, taxes, bonuses, incentive bonuses, etc. These payments themselves already contain a qualitative and quantitative assessment of the work of employees.

Under the cost of human capital to increase the level of the intellectual capital of the enterprise means the cost of training, retraining, training of employees, participation in thematic seminars, conferences, symposia; costs of research, information, and legal systems, software, literature, including periodicals, etc.

These costs, investments in HC are aimed at innovative reproduction of intellectual capital, to increase the intellectual level of employees, which in turn allows more efficient use of available limited human resources in accordance with the needs of the enterprise.

The cost of health capital is an investment in a person, made in order to form, maintain, and improve his health and efficiency, aimed at increasing the capital of the culture of possession of information. Such costs include health care costs (preventive examinations of employees, additional health insurance, spa treatment, and other disease prevention and control measures), as well as costs aimed at improving the safety of workers places, organization of sports leisure of employees, improvement of living conditions, etc. The main thing in this is the awareness of the individual that his health and culture of behavior is an opportunity to replenish the information potential of activity in making informed decisions. That is, investment in the personality mentality is an investment in the culture of the individual, which determines the efficiency and accelerates the growth of human capital.

In formal form, the expression for quantifying HumanCap's human capital will look like an additive model representing the sum of five components:

$$
\begin{aligned}
\text { HumCap }=W F+I n t C a p & + \text { HealCap }+ \\
& + \text { CultCap }+ \text { InfCap },
\end{aligned}
$$

where $W P$ - remuneration of personnel of the enterprise to the general fund of remuneration;

IntCap - the share of costs in human capital to increase the level of the intellectual capital of the enterprise:

$$
\text { IntCap = InvestCap } / \text { TotalExp, }
$$

where: InvestCap - investment in the HC for innovation reproduction of the intellectual capital;

TotalExp - the total cost of the company to increase $\mathrm{HC}$;

HealCap - the share of costs (investments) of the enterprise for health capital,

$$
\text { HealCap }=\text { HealthReprod } / \text { TotalExp, }
$$

where: HealthReprod - the cost (investment) to reproduce the health of staff;

CultCap - the company's cost of capital culture of information ownership:

$$
\text { CultureCap }=\text { CultureKnowH } / \text { TotalExp, }
$$

where: CultureKnowH - the share of personal spending on KnowHow and possession of information and the ability to use it;

InfCap - enterprise costs for information capital,

InformCap $=$ InformConvert $/$ TotalExp,

where: Inform Convert - the cost of the individual's ability to turn information into the necessary knowledge of the company, which is of particular importance for its activities.

Keep in mind that each cost element has its impact.

For example, the return on investment in intellectual capital is higher than on investment in "health capital". Therefore, it is advisable to introduce weighting factors - for each type of cost.

$$
\begin{aligned}
& \text { HumCap }=W F \alpha_{1}+\operatorname{IntCap} \alpha_{2}+ \\
& \text { HealCap } \alpha_{3}+\text { CultCap } \alpha_{4}+\operatorname{InfCap} \alpha_{5},
\end{aligned}
$$

where $\alpha_{1}$ - capital of competence "property-competence";

$\alpha_{2}$ - educational capital "property of creativity";

$\alpha_{3}$ - health capital "property-motivation";

$\alpha_{4}-$ culture capital "property-purpose";

$\alpha_{5} \quad-$ the information capital "propertycommunicative".

The value of the coefficients is assigned based on the value of the return of each component in the $\mathrm{HC}$ of the enterprise. In determining the return on each type of cost must take into account the following, most important factors:

1. The industry in which the company operates.

2. The degree of "intellectualization" of labor, the level of knowledge-intensive products.

3. Stage of the life cycle of the enterprise.

4. The presence of a system for assessing the intellectual level of staff and its motivation.

5. Culture of information ownership.

6. Investments in human capital for innovations in the reproduction of intellectual capital. 


\section{\begin{tabular}{|l|l|}
\hline \multicolumn{2}{|c|}{ Intellectual capital } \\
\hline Consumer capital & Organizational capital \\
\hline
\end{tabular}}

Human capital - HC
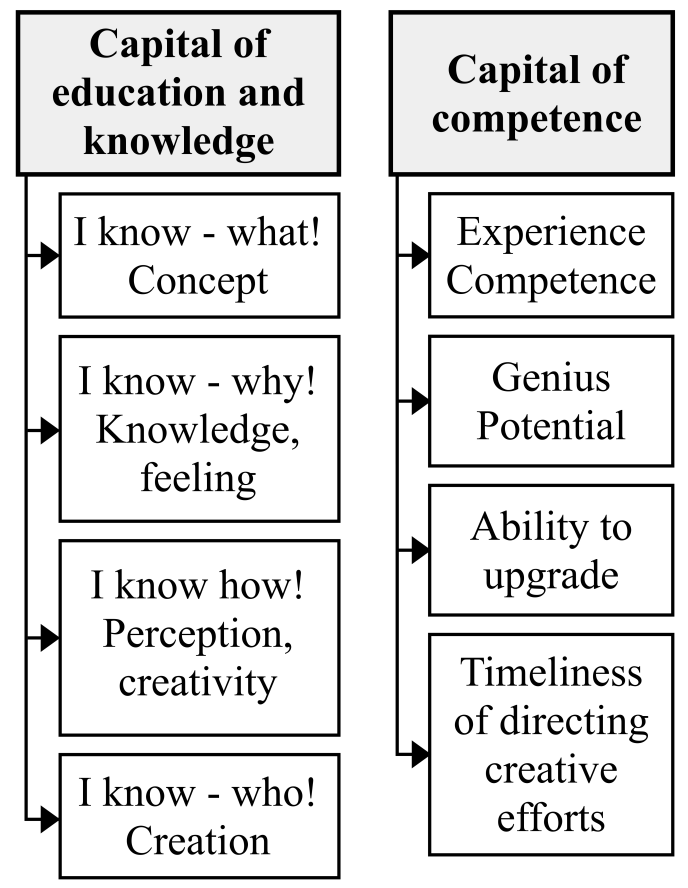

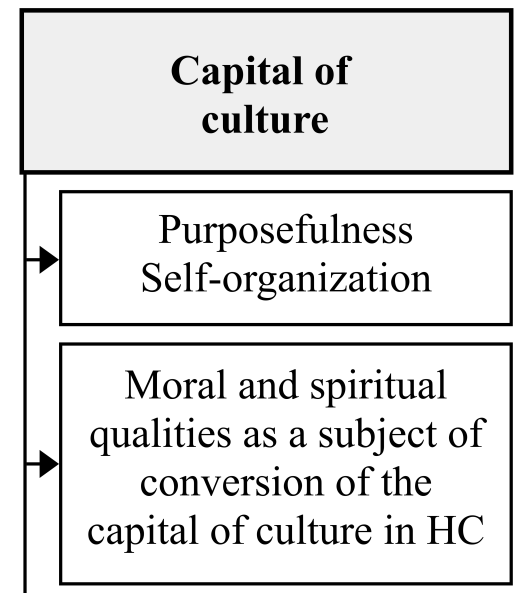

The ability to make meaningful perceptions

of interdependent relationships between actors
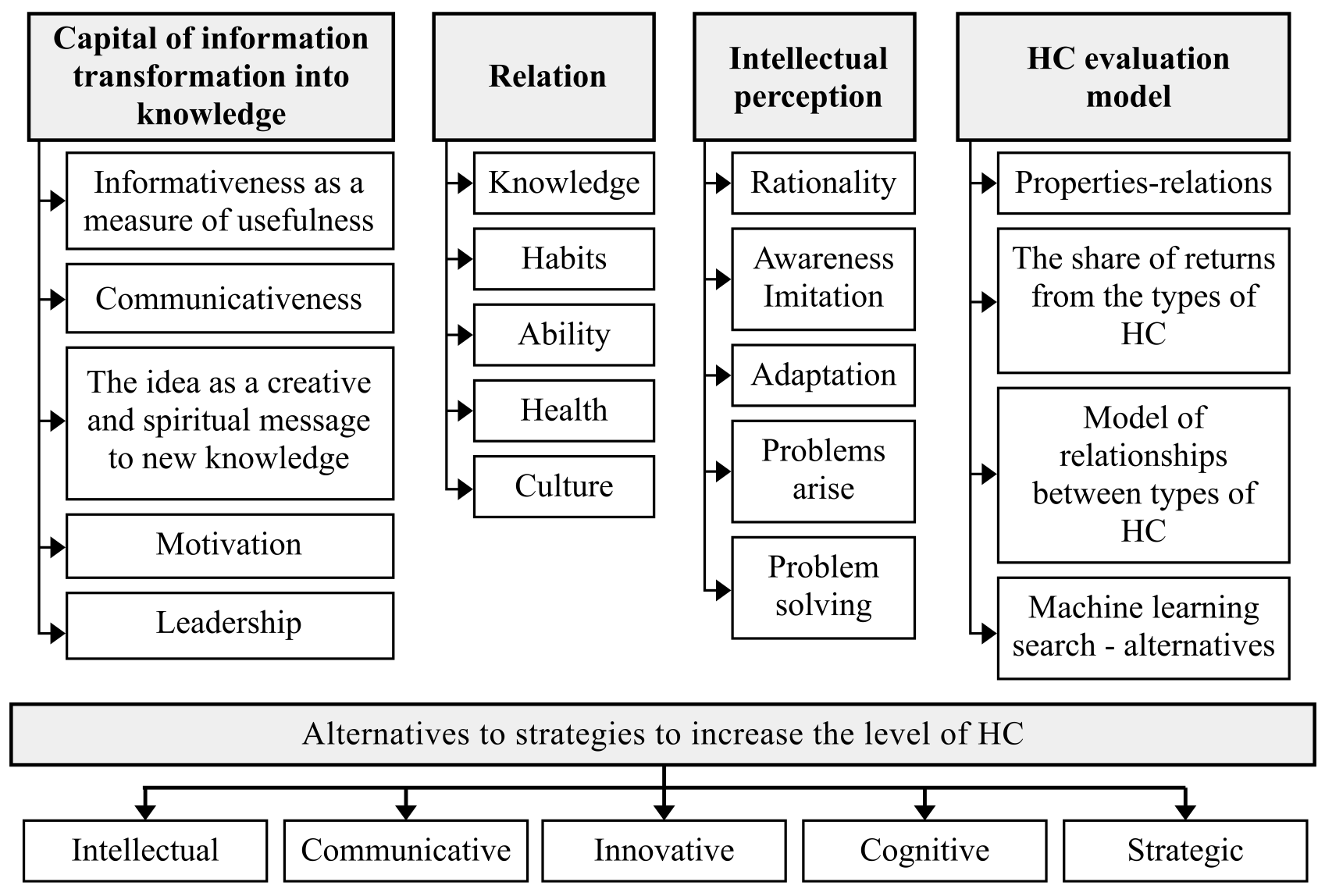

Figure 1. Human capital 
7. The cost of the ability of the individual to turn information into the necessary knowledge of the company.

In addition, investment in human capital for innovation reproduction of intellectual capital and company health is influenced by concepts such as secondary education and their average age. The dependence of the efficiency of intellectual capital costs on the general level of education, the cost of health capital on the average age of staff can be taken into account through the parameters $\varepsilon_{1}$ and $\varepsilon_{2}$ respectively.

$$
\begin{aligned}
\text { WF } \alpha_{1}=\operatorname{IntCap} \alpha_{2} \varepsilon_{1} & + \text { HealCap } \alpha_{3} \varepsilon_{2}+ \\
& + \text { CultCap } \alpha_{4}+\text { InfCap } \alpha_{5},
\end{aligned}
$$

where $\varepsilon_{1}, \varepsilon_{2}$-parameters that reflect the dependence of cost recovery on the level of education and age, respectively.

$$
\varepsilon_{1}=\frac{\sum_{i=1}^{n} r_{i} z_{i}}{R_{S} z},
$$

where $r_{i}$ and $z_{i}$-the number of employees with i-level of education and their average salary;

$n$ - the number of levels of education in a particular enterprise;

$R_{S}$ - the total number of personnel of the enterprise;

$z$ - the average salary of employees of the basic level of education.

The function that reflects the dependence of the return on health capital costs is as follows:

$$
\varepsilon_{2}=\gamma_{m} \sum_{j=1}^{n} k_{j} d_{j}+\gamma_{w} \sum_{j=1}^{n} k_{j} t_{j},
$$

where $\gamma_{m}, \gamma_{w}$ - the share of men and women, respectively, in the enterprise;

$d_{j}, t_{j}$ - the share of employees of men and women of the $j$-age group;

$n$ is the number of age groups;

$k_{j}$ - rate of return on investment in health capital depending on age groups, which are determined on the basis of retirement age. Appropriate coefficient values are assigned to each age group.

Coefficients of return on investment depending on the components of human capital are characterized by cognitive properties (creativity, competence, purposefulness, communication, motivation), between varieties $(S)$ in which there are appropriate reflexive relations $(\alpha)$ of their interaction, figure 2 [5].

Thus, the model for assessing the level of a particular type of specific property of human capital and its quantitative assessment is determined by the formula:

$$
H C=\alpha_{i}\left(\sum_{t} S_{i p}^{z} \times \text { HumanCap }_{i}\right),
$$

where $S_{i p}^{z}=\sum_{j=1}^{n_{i p}} k_{j p}^{a} * \alpha_{i j p}^{z}$ - the level of the $i$ variety of the $p$ property;

$\alpha_{i j p}^{z}$ - the share of participation of the $j$ relation in the formation of the $i$ variety of the $p$ property of the $z$ individual;

\begin{tabular}{|l|l|}
\hline Creativity & $S_{1}$ \\
\hline Competence & $S_{1}$ \\
\hline Pursefulness & $S_{1}$ \\
\hline Communicative & $S_{1}$ \\
\hline Motivation & $S_{1}$ \\
\hline$S_{I}$ & Health \\
\hline$S_{I}$ & Culture \\
\hline
\end{tabular}

Figure 2. The properties and reflexive relations of human capital

$k_{j p}^{a}$ - the basic coefficient of distribution of the influence of the $j$ relation on the formation of the $p$ property;

$n_{i p}$ - the number of relations of the $i$ variety of the $p$ property;

$S_{i p}^{z}$ - the level of the $i$ variety of the $p$ property;

HumanCap - human capital of the species.

The level of significance of the criteria is determined by the methodology of the MAI (table 1).

Table 1. The level of the weight of the criteria

\begin{tabular}{ll}
\hline Criterion & Result \\
\hline Creativity & 0.223 \\
Competence & 0.244 \\
Purposefulness & 0.2 \\
Communicative & 0.189 \\
Motivation & 0.144 \\
\hline
\end{tabular}

Analysis of the criteria, their weight, allows you to choose the best alternative for the development of the properties of human capital. To do this, use the method of hierarchical comparisons in assessing the level of prior-

\begin{tabular}{|c|c|c|c|c|c|}
\hline \multirow[t]{2}{*}{ Criterion } & \multicolumn{5}{|c|}{ Properties } \\
\hline & 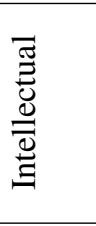 & 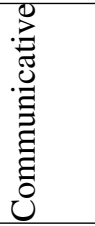 & 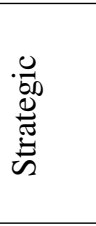 & ن̊. & 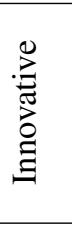 \\
\hline Creativity & 0.216 & 0.172 & 0.207 & 0.172 & 0.23 \\
\hline Competence & 0.208 & 0.2 & 0.189 & 0.226 & 0.17 \\
\hline Purposefulness & 0.176 & 0.177 & 0.27 & 0.19 & 0.18 \\
\hline Motivation & 0.168 & 0.19 & 0.189 & 0.2 & 0.26 \\
\hline Communicative & 0.164 & 0.227 & 0.182 & 0.23 & 0.2 \\
\hline $\begin{array}{l}\text { General } \\
\text { proach }\end{array}$ & 0.189 & 0.195 & 0.208 & 0.202 & 0.21 \\
\hline
\end{tabular}
ities of alternatives, the results of which are given in the table 2 and in figure 3 .

Table 2. Influence of criteria on a choice of alternatives (properties) of improvement of the level of human capital

The process of investing in human capital should be divided into several stages of investing: education costs; the cost of finding and hiring staff; staff costs during the training period; staff costs during the period of accumulation 


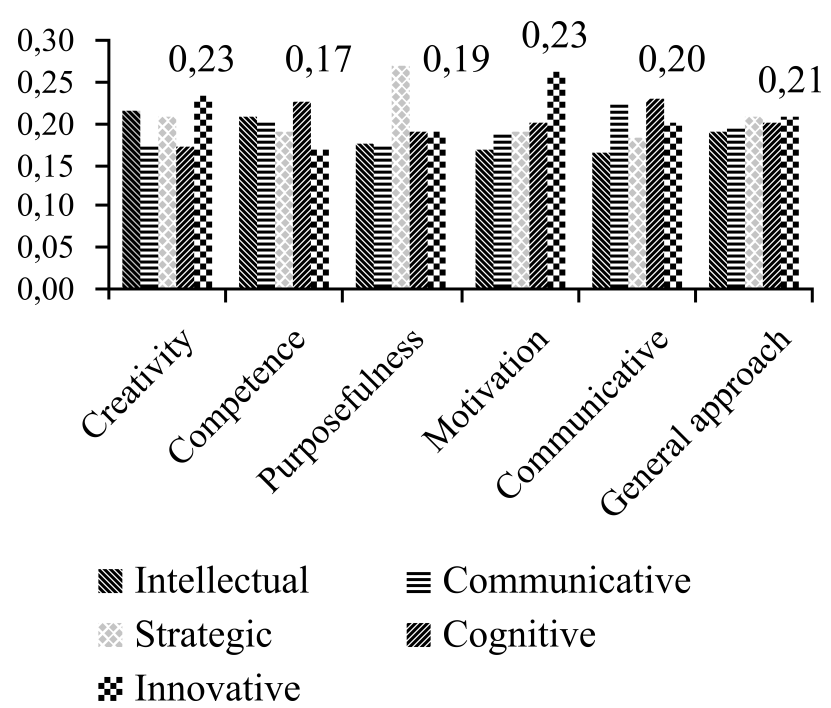

Figure 3. The influence of criteria on the choice of alternatives

of growth potential; staff costs during the period of professionalism; staff costs during training, advanced training; staff costs in the period of decline and "obsolescence" of professionalism; investment in human capital for innovations in the reproduction of intellectual capital; the cost of the individual's ability to turn information into the necessary knowledge companies; the cost of information culture.

The development entity itself can invest in its personal human capital, but these investments can be formed by the enterprise, to increase the human capital of the organization or the state, to increase the national human capital.

Based on the assessment of investment in human capital, the assessment of the direct economic effect is carried out. Such an assessment is possible, as a rule, only after the implementation of any investment project involving staff. To assess the economic effect is more suitable known ROI (Return on Investment):

$$
R O I=(\text { OutInv }- \text { PostInv })-Z) / Z 100 \%,
$$

where OutInv - the cost of the human capital of the individual before training;

PostInv - the cost of the human capital of the individual after receiving educational services;

$Z$ - direct and indirect costs of the investment project.

The value of ROI is crucial in measuring the effectiveness of human capital development strategy. Thus, if the value of ROI is less than $20 \%$, the development strategy is considered ineffective, in the case of around $20 \%$, we can say that the company is developing moderately, and sometimes completely stopped in development. A good indicator of development is a value of $150 \%$ to $200 \%$, and anything between $20 \%$ and $150 \%$ can be called an effective strategy.

The relevance of the use of machine learning in the field of economics allows us to consider differently many aspects of human capital development strategies. Training to find the best strategy for human capital development can be represented as a continuous cycle, which will end only after reaching the specified conditions (figure 4).

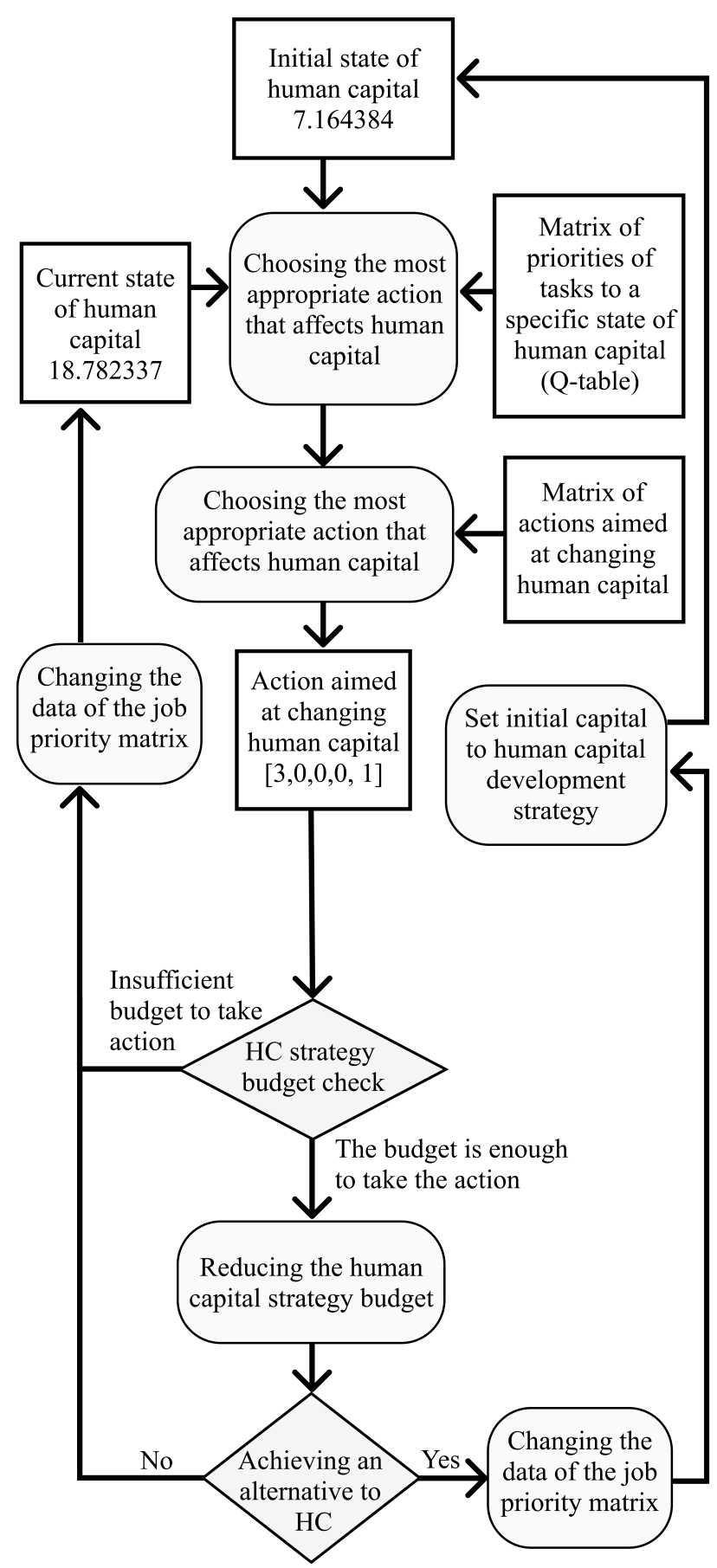

Figure 4. Machine learning of alternative development of human capital of the enterprise

In the reinforcement learning algorithm, the agent's actions are directed to the steps to achieve success with a reward estimate. After $\Delta t$ steps into the next step, the human capital will decide some next step. The weight for this step is calculated as $\gamma^{\Delta t}$, where $\gamma$ is the discount factor, which can take a value from 0 and $1,0 \leq \gamma \leq 1$ and has the effect of evaluating actions that are aimed at achieving the human capital goal. $\gamma$ can be called the level of success 
in achieving the desired state by human capital, when the investment data changes at the $\Delta t$ step.

Thus, we can conclude that a function is required that will determine the quality of combinations of the state of human capital and the action aimed at it:

$$
Q \div S \times A \rightarrow R
$$

At the beginning of training, $Q$ is initialized, possibly with an arbitrary fixed value -0 . After initialization, at each moment of time $t$, the agent selects an action, observes a reward, enters a new state (that may depend on both the previous state and the selected action), and $Q$ is updated. The core of the algorithm is a Bellman[6] equation as a simple value iteration update, using the weighted average of the old value and the new information [7]:

$$
\begin{aligned}
Q^{\text {new }}\left(s_{t}, a_{t}\right) \leftarrow & Q\left(s_{t}, a_{t}\right)+\alpha \times \\
& \left(r_{t}+\gamma \times \max Q\left(s_{t+1}, a\right)-Q\left(s_{t}, a_{t}\right)\right),
\end{aligned}
$$

where $r_{t}$ is the reward received when moving from the state $S_{t}$ to the state $S_{t+1}$, and $0<\alpha \leq 1$.

Note that $S^{\text {new }}\left(s_{t}, \alpha_{t}\right)$ is the sum of three factors:

1) $(1-\alpha) Q\left(s_{t}, \alpha_{t}\right)$ : the current value weighted by the learning rate. Values of the learning rate near to 1 made faster the changes in $Q$;

2) $\alpha r_{t}$ : the reward $r_{t}=r\left(s_{t}, a_{t}\right)$ to obtain if action $a_{t}$ is taken when in state $s_{t}$ (weighted by learning rate);

3) $\alpha \gamma \max Q\left(s_{t+1}, \alpha\right)$ : the maximum reward that can be obtained from state $s_{t+1}$ (weighted by learning rate and discount factor).

The episode of the algorithm ends when the state $S_{t+1}$ of human capital is the desired one or the financial investment opportunities end. However, Q-learning can also be trained on non-ad-hoc tasks. If the discount factor is below 1 , the action values are finite, even if the problem may contain infinite loops.

For all final states $s_{f}, Q\left(s_{f}, \alpha\right)$ is never updated but is set to the reward valuer observed for state $s_{f}$. In most cases, $Q\left(s_{f}, \alpha\right)$ can be taken to equal zero.

Before starting training according to the Q-learning method to find the best development strategy, the parameters of the current human capital are determined. The result of the calculations will be the state of human capital in the q-table. At the beginning of training, the entire matrix of the priority of the choice of actions aimed at the development of human capital will be equal to zero. If all the priorities or some of them are equal, then the choice is made randomly.

After choosing an action and applying it, we recalculate the value of human capital to determine a new state. Actions, in this case, are aimed at increasing the costs of one or more categories of human capital, table 3.

The success of performing actions is a certain human capital to which the subject strives. However, it is worth considering financial constraints, which may vary depending on the situation. For example, health costs for a specific activity cannot be less than $20 \%$ of total costs. Then, even if the desired value of human capital is obtained and modeled, but the condition for health capital is not met, then the learning cycle is considered ineffective. Limitations may also be on the part of the budget if any, but in some cases, with a budget limitation, the specified goal of human capital may not be achieved.

Table 3. Q-Learning state table for actions aimed at changing the values of human capital criteria, through training, when each cell is updated through training

\begin{tabular}{cccc}
\hline \multirow{2}{*}{$\begin{array}{c}\text { The state } \\
\text { of human } \\
\text { capital }\end{array}$} & \multicolumn{3}{c}{$\begin{array}{c}\text { Actions aimed at changing } \\
\text { human capital }\end{array}$} \\
\cline { 2 - 4 } & $\begin{array}{c}\text { Enhancing } \\
\text { creativity }\end{array}$ & $\begin{array}{c}\text { Health } \\
\text { Capital } \\
\text { Gain }\end{array}$ & $\begin{array}{c}\text { Boost } \\
\text { motivation }\end{array}$ \\
\hline 13.435367 & $-11,435600$ & $-84,758326$ & $-54,23453$ \\
$\ldots$ & $\ldots$ & $\ldots$ & $\ldots$ \\
18.782337 & $-2,543467$ & $-3,246473$ & $-26,26434$ \\
$\ldots$ & $\ldots$ & $\ldots$ & $\ldots$ \\
180.28351 & $-8,546123$ & $-30,245365$ & $-2,234563$ \\
\hline
\end{tabular}

If you do not limit the total budget, then after going through the training cycle and receiving the optimal algorithm for the development of human capital, at same time you can find out the required budget for the implementation of this strategy

Having completed the training, we get a filled matrix, where the rows are the states of human capital, and the columns are possible actions (table 4) aimed at human capital.

Table 4. Matrix of actions aimed at changing the parameters of

\begin{tabular}{|c|c|c|c|c|c|}
\hline \multirow[b]{2}{*}{ 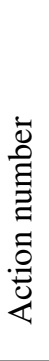 } & \multicolumn{5}{|c|}{$\begin{array}{l}\text { Impact values } \\
\text { on human capital }\end{array}$} \\
\hline & 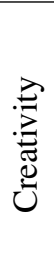 & 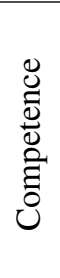 & 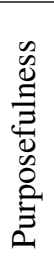 & 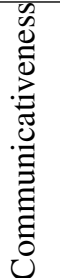 & 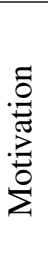 \\
\hline 1 & 12 & 0 & 0 & 0 & 0 \\
\hline 2 & 3 & 0 & 4 & 0 & 0 \\
\hline 3 & 0 & 0 & 0 & 7 & 2 \\
\hline 4 & 1 & 1 & 1 & 0 & 0 \\
\hline 5 & 0 & 0 & 21 & 0 & 0 \\
\hline 6 & 0 & 0 & 0 & 0 & 32 \\
\hline
\end{tabular}
human capital with indicators of the impact on human capital and the required budget

The optimality of using machine learning in this method lies in the speed of finding a sequence of actions that affect the change in human capital and its structure. With its help, knowing the initial data and the planned budget for the development of human capital, it is possible to optimize costs, taking into account the specifics of the subject, whose human capital is being considered. That is, having the same budget, using machine learning, you can 
calculate the most effective strategy for the development of human capital.

The main difficulty of this approach to the choice of alternative solutions for the search for options for using human capital is the correct selection of the coefficients of the significance of the contributions, on the basis of which the learning cycles of the system will take place. This approach can simplify the creation of a strategy for human capital development, present alternative ways, and simplify management decisions, table 5 .

Table 5. Initialized data affecting machine learning training to find optimal investments in human capital without the limitations of the human capital development fund

\begin{tabular}{|c|c|c|c|c|c|c|}
\hline \multicolumn{5}{|c|}{$\begin{array}{l}\text { Criteria Properties } \\
\text { (One hundredth part) }\end{array}$} & \multirow[b]{2}{*}{ 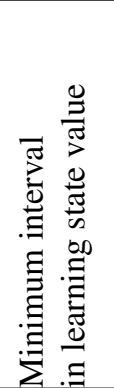 } & \multirow[b]{2}{*}{ 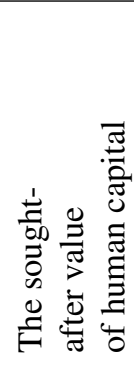 } \\
\hline 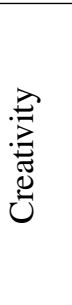 & 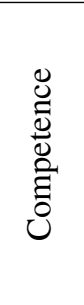 & 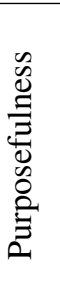 & 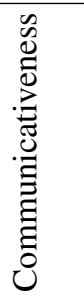 & 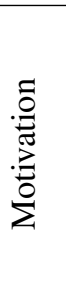 & & \\
\hline 197 & 225 & 158 & 182 & 212 & 0.0001 & 25 \\
\hline
\end{tabular}

After making calculations with the initial data presented in table 6, results were obtained that describe the strategy of investing in human capital.

It should be borne in mind that the coefficients of the importance of each of the parameters do not allow a decrease in investment for each of the parameters to zero. The input data for training are: the coefficients of the importance of each of the categories and the required value of human capital.

Table 6. Initialized data affecting machine learning training to find optimal investments in human capital without the limitations of the human capital development fund.

\begin{tabular}{|c|c|c|c|c|c|c|}
\hline \multirow[b]{2}{*}{ 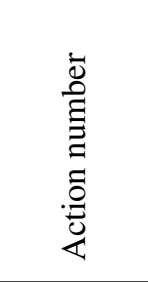 } & \multicolumn{6}{|c|}{ Impact values on human capital } \\
\hline & $\frac{\vec{E}}{\tilde{E}}$ & 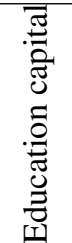 & 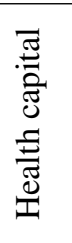 & 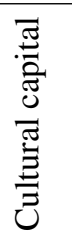 & 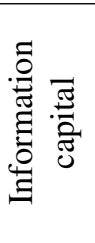 & 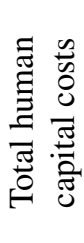 \\
\hline 15637 & 219 & 188 & 630 & 260 & 22 & 1519 \\
\hline 21352 & 114 & 155 & 121 & 95 & 128 & 621 \\
\hline 71521 & 103 & 157 & 151 & 61 & 129 & 601 \\
\hline 128868 & 110 & 196 & 115 & 72 & 103 & 596 \\
\hline 2034991 & 158 & 156 & 87 & 50 & 136 & 587 \\
\hline 11000000 & 106 & 197 & 90 & 51 & 141 & 585 \\
\hline 13243567 & 136 & 187 & 89 & 50 & 122 & 584 \\
\hline
\end{tabular}

The results of the strategy of investing in human capital are highly dependent on the learning cycle. So on very small training cycles, investment is very inconsistent, but in the next step, although the investment funds change their direction, it is more and more difficult to achieve a stronger human capital with less investment.
The data in table 6 and figure 5 show the optimization of the costs of human capital development to achieve a human capital value of 25 units. It can be concluded that in order to obtain better results, it is necessary to carry out a sufficient number of training cycles. You can see how, when setting the initial data, the system gives priority to the wrong criteria for investment, which have become priority in recent cycles.

Taking into account the dynamics of changes in results, it can be concluded that subsequent training cycles can bring more optimized costs.

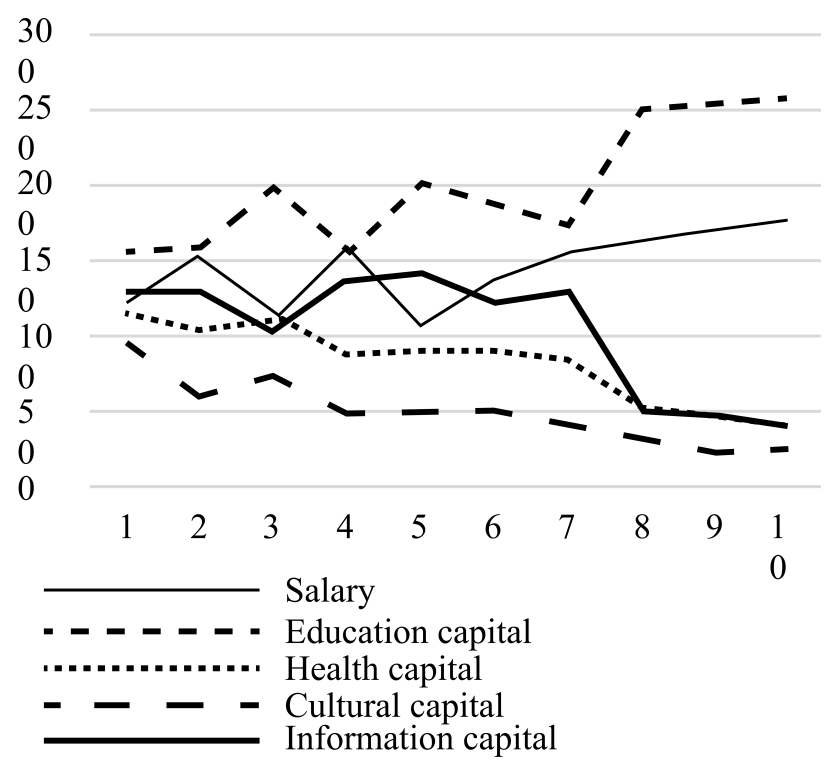

Figure 5. Machine learning of alternative development of human capital of the enterprise

Fig. 6 shows the optimization of the costs of human capital development, taking into account the same level of human capital development.

After training the parameters of the model, we determine the new values of the return coefficients for each type of human capital. As a result, the parameters of the alternatives that determine the planning of new investments in the HC structure change, which entails the search and recalculation of new behavior.

Thus, after each stage of training, new indicators of alternatives should be determined and calculations should be made that determine the subsequent investments in human capital. It should also be borne in mind that each alternative has its own characteristic features and properties of behavior and influence on the choice of options for investing in capital. Making decisions aimed at assessing the interaction of the relationship of these properties in the capital structure can change cognitive and reflexive attitudes and affect the level of human capital.

It should also be borne in mind that the definition of new properties in terms of alternatives can give us information about an insufficient assessment of the interaction of the relationships of these properties in the capital structure. That entails adjusting the choice of options and the redistribution of investment in the capital structure. 
The results of changes in human capital allow us to determine the return of its structural elements of human capital. Redistribution of coefficients alternatives should be redistributed taking into account these data. The current situation requires development in the direction of choosing alternatives of a cognitive, strategic and innovative type.

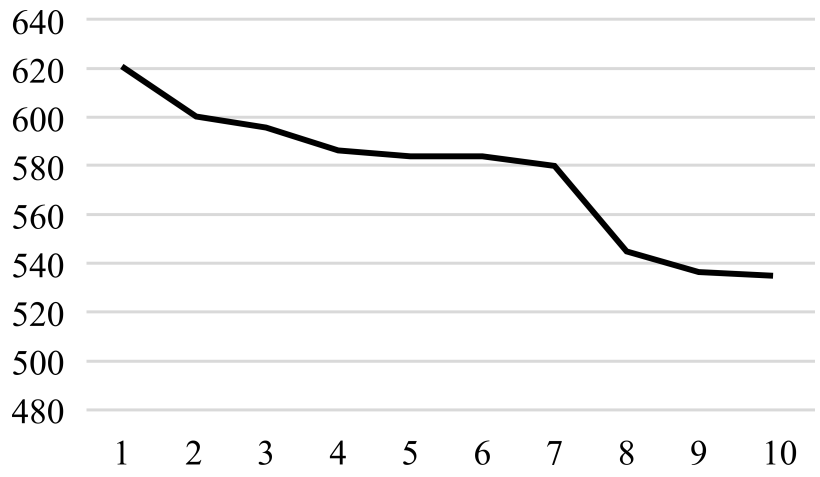

Figure 6. Machine learning of alternative development of human capital of the enterprise

Is a promising approach to modeling intellectual capital in general. It is also worth noting that when the input data changes, machine learning will be able to rebuild and perform calculations and optimize the result better and faster than a human.

\section{Conclusions}

As a result of research, the concept of a flexible evolutionary model is proposed, which with the help of machine learning allows obtaining the most successful strategy for the development of human capital. The proposed conceptual and methodological approach to machine learning of the process of assessing human capital of enterprises, taking into account the cognitive psychology of man and reflective attitudes in the human environment, can increase the effectiveness of decision-making in the field of human capital development management.

The training involves indicators of return on investment in the individual, in the types of components of human capital, which are characterized by properties (creativity, competence, purposefulness, communication, motivation), where between their varieties there are reflective relationships.
The main difficulty of this approach to the choice of alternative solutions for finding options for the use of human capital is the correct selection of indicators of significance (return) of contributions to the development of types of human capital, on the basis of which cycles occur of systemic learning.

This approach can simplify the search for and developments of human capital development strategies, present alternative ways, and simplify management decisions.

It is worth noting that setting up training with changing training parameters, namely the sum of the reward and the value of data optimization, can achieve better results by accelerating training, and therefore obtaining data on a more trained AI, which can give better results.

Using machine learning to optimize the cost of human capital development is a better method. Speed, lack of subjectivity, and the ability to quickly respond to external changes is an advantage over a person.

This method has a number of possible improvements. For example, it is worth considering the obsolescence of some of the capitals. Also, one of the criteria is wages and its impact on human capital depends on the return, but it should be borne in mind that low returns should not always affect the decrease in this parameter. Also, this method allows, using other input parameters and the limit in the human capital development fund, to find out the development limit and also optimize the costs for each of the human capital funds.

\section{References}

[1] L. Yang, H. Xu, Y. Jiang, Procedia Computer Science 131, 1282 (2018)

[2] F. Fallucchi, M. Coladangelo, R. Giuliano, E. William De Luca, Computers 9 (2020)

[3] T.W. Schultz, Human Capital: Policy Issues and Research Opportunities (UMI, 1972), pp. 1-84, https: //core.ac.uk/download/pdf/6908045.pdf

[4] G.S. Becker, Human Capital: A Theoretical and Empirical Analysis, with Special Reference to Education, 3rd edn. (University of Chicago Press, 1994)

[5] V.M. Porohnya, Intellectual Capital of Economic Growth: A Manual (CPU, 2012)

[6] R.E. Bellman, Dynamic Programming (Princeton University Press, New York, 1957)

[7] C.J.C.H. Watkins, P. Dayan, Machine Learning 8, 279 (1992) 